\title{
PEPRINE

\section{5) Produtos de glicação avançada, capacidade funcional e cognitiva em portadores da Doença de Parkinson}

Jenifer Kristina A. de Almeida'; Natalia Mariana Silva Luna²; Rodrigo Tallada Iborra; Angélica Castilho Alonso ${ }^{3}$; Adriana Machado-Lima ${ }^{3}$

\section{Resumo}

Pode ocorrer na Doença de Parkinson o aumento da formação de proteínas agregadas pela glicação, o que contribui para o declínio da capacidade funcional e cognitiva dos pacientes.

Palavras-chave: Produtos de glicação avançada, Doença de Parkinson, Envelhecimento, Cognição e Desempenho motor.

\section{Introdução}

A glicação parece aumentar a formação de proteínas agregadas durante a Doença de Parkinson (DP), contribuindo para o declínio da capacidade funcional e cognitiva dos pacientes com DP.

\section{Objetivo}

Estimar o consumo e a formação dos produtos de glicação avançadas (AGE) em indivíduos portadores de Parkinson e relacionar com sua capacidade funcional e função cognitiva.

1 Mestre pelo Programa de Pós-graduação Ciências do Envelhecimento da Universidade São Judas Tadeu, SP. Endereço para correspondência: Rua Taquari, 546 - Mooca, São Paulo - SP CEP 03166-000 Tel.: 2799-1944. E-mail: jenifer_ kr@hotmail.com

2 Professora na Universidade Anhembi Morumbi. E-mail: nmsluna@gmail.com

3 Professores do Programa de Pós-graduação Ciências do Envelhecimento da Universidade São Judas Tadeu. E-mails (na ordem em que aparecem): rtiborra@yahoo.com.br; angelicacastilho@msn.com; prof.adrianalima@usjt.br 


\section{Método}

O trabalho foi aprovado pelo Comitê de Ética em Pesquisa da Universidade São Judas Tadeu (CAAE: 79991417.9.0000.0089). Foi realizada uma pesquisa com 20 portadores da DP e 20 indivíduos controles de ambos os sexos com mais de 55 anos. Foi utilizado leitor de autofluorescência para medir o AGE da pele e foi aplicado recordatório alimentar de três dias distintos para estimar o consumo de AGE. O Montreal Cognitive Assessment (MoCA) foi aplicado para análise da função cognitiva, além do Short Physical Performance Battery (SPPB) e Força de Preensão Manual para avaliação da capacidade funcional. As análises estatísticas foram feitas com auxílio do Software Graph Pad Prism 6.0. Foi feito a aplicação do teste de normalidade Kolmogorov-Smirnov, e seguiu-se com correlação de Pearson ( $\mathrm{p}<0,05)$.

\section{Resultados}

Não houve diferença da capacidade cognitiva entre os grupos. Pacientes com DP apresentam pior capacidade funcional relacionado à força, ao equilíbrio e à marcha, quando comparados aos indivíduos controles. Indíviduos com DP apresentaram uma tendência a consumir menor quantidade de AGE (18311 $\pm 6458 \mathrm{kU} / \mathrm{d})$ quando comparado ao grupo controle $(24544 \pm 13384 \mathrm{kU} / \mathrm{d}), \mathrm{p}=0,07$. Indivíduos com DP que consomem mais AGE apresentaram menor força $(p=<0,05 ; r=-0,26)$.

\section{Conclusão}

A despeito da menor formação e consumo de AGE, indivíduos com DP que ingerem mais AGE apresentaram menor força e consequentemente piora da capacidade funcional, sugerindo então que os efeitos dos AGEs podem ser exacerbados no organismo na vigência de uma doença crônica, como o Parkinson.

\section{Advanced glycation products, functional and cognitive capacity in patients with Parkinson's disease}

\section{Abstract}

Parkinson's disease may increase the formation of proteins aggregated by glycation, which contributes to the decline of functional and cognitive capacity of patients.

Keywords: Advanced glycation products, Parkinson's disease, Aging, Cognition and Motor performance. 